\title{
A rare case of severe gastroenteritis caused by Aeromonas hydrophila after colectomy in a patient with anti-Hu syndrome: a case report
}

\author{
Michael Greiner ${ }^{1}$, Alexia Anagnostopoulos ${ }^{2}$, Daniel Pohl ${ }^{3}$, Reinhard Zbinden ${ }^{1}$ and Andrea Zbinden ${ }^{*}$ (i)
}

\begin{abstract}
Background: Aeromonas hydrophila is a gram-negative facultative anaerobic coccobacillus, which is an environmental opportunistic pathogen. A. hydrophila are involved in several infectious diseases such as gastroenteritis, septicemia and wound infections. However, gastroenteritis caused by Aeromonas spp. are rare and the clinical relevance of Aeromonas species in stool specimens is still under debate.
\end{abstract}

Case presentation: Our case concerns a 32-year-old woman who presented at hospital with a worsening watery diarrhea and fever requiring intensive care. A cholera-like illness was diagnosed. The patient had a past history of an anti-Hu syndrome with a myenteric ganglionitis. A molecular multiplex RT-PCR (QIAstat-Dx Gastrointestinal Panel, QIAGEN) covering a broad spectrum of diverse gastrointestinal pathogens performed directly from the stool was negative but the stool culture revealed growth of A. hydrophila. Further investigations of the A. hydrophila strain in cell cultures revealed the presence of a cytotoxic enterotoxin.

Conclusions: Although A. hydrophila rarely causes gastroenteritis, Aeromonas spp. should be considered as a causative agent of severe gastroenteritis with a cholera-like presentation. This case highlights the need to perform culture methods from stool samples when PCR-based methods are negative and gastrointestinal infection is suspected.

Keywords: Aeromononas hydrophila, Gastroenteritis, Anti-Hu syndrome, Cytotoxic enterotoxin, Case report

\section{Background}

Aeromonas spp. can cause different clinical diseases especially in the immunocompromised host. The most common infection sites are wound infections, cellulitis, septicemia and urinary tract infections [1-4]. Gastroenteritis due to Aeromonas spp. is generally rare but has been described before in the literature [5-7]. The clinical presentation of gastroenteritis varies from mild diarrhea

\footnotetext{
${ }^{*}$ Correspondence: azbinden@imm.uzh.ch

${ }^{1}$ Institute of Medical Microbiology, University of Zurich, Gloriastrasse 28/30, 8006 Zurich, Switzerland

Full list of author information is available at the end of the article
}

to shigella-like dysentery to severe cholera-like watery diarrhea [8].

Aeromonas spp. are gram-negative facultative anaerobes that are straight, coccobacillary to bacillary cells with rounded ends. Aeromonads usually are oxidase positive and display a fermentative metabolism of glucose. The organisms grow at a range of temperatures from 10 to $42{ }^{\circ} \mathrm{C}$ [4]. The genus Aeromonas currently consists of 36 species, of which Aeromonas hydrophila subsp. hydrophila, Aeromonas caviae, Aeromonas dhakensis, Aeromonas veronii biovar sobria (formerly Aeromonas sobria) and Aeromonas trota are clinically most important and have been isolated often from human feces [9]. 
Aeromonads feature several virulence factors such as the cytotoxic enterotoxin (Act protein), which has hemolytic, cytotoxic, and enterotoxic activities; type 3 secretion systems and motility factors [10-13]. Enterotoxins play a relevant role in the pathogenesis of diarrhea and their effect is reproducible in animal models [14]. The cytotoxic enterotoxin Act was previously isolated and extensively characterized [15]. Recently, there were also two cytotonic enterotoxins described, a heat stable (Ast protein) and a heat labile enterotoxin (Alt protein) [16, 17]. The presence of Ast and Alt cytotonic enterotoxins in Aeromonas spp. were associated with severe diarrhea in children, however, these toxins were found also in environmental strains [16]. To our knowledge, the enterotoxins are chromosomally encoded $[10,11,18]$; the role of plasmids are unknown with the exception of one reported case with evidence of a Shiga-like toxin 1 on a plasmid in strains of $A$. hydrophila [10-13].

The role of Aeromonas spp. as enteropathogen is still controversial [19]. We report on a case where severe watery diarrhea was caused by $A$. hydrophila resulting in intensive care medical occupancy. We investigated phenotypically whether the clinical strain isolated from feces produced a cytotoxic enterotoxin.

\section{Case presentation}

A 32-year-old female patient was admitted to hospital with somnolence, aggravation of her chronic diarrhea and fever. The patient had a complicated medical history of intestinal neuronopathy with recurrent pseudoobstructions due to myenteric ganglionitis. The patient had therefore undergone a hemicolectomy and permanent jejuno-rectostomy. She then developed chronic diarrhea (3 to 4 times per day), which led to a chronic hyponatremia (125 mmol/l, normal value between 135$145 \mathrm{mmol} / \mathrm{l})$. Less than a month prior to hospital admission, anti-Hu antibodies were detected associated with paraneoplastic neurological syndromes [20,21]. An anti$\mathrm{Hu}$ syndrome with sensory neuronopathy was diagnosed. Despite extensive diagnostics, no underlying malignancies could be found. Immunosuppressive therapy had not yet been initiated.

One week before the current presentation at the hospital, she developed fever, chills, abdominal pain and an increased stool frequency (up to 10 times a day). The diarrhea was of watery consistency without blood or mucus. She had no contact to animals and did not consume contaminated food. None of her social contacts had signs of infectious gastroenteritis. At the emergency room, she was febrile $\left(39.5^{\circ} \mathrm{C}\right)$ and abdominal examination revealed increased bowel sounds without tenderness on palpation. She was somnolent without focal neurological deficits. The laboratory studies showed elevated inflammatory markers $(157 \mathrm{mg} / \mathrm{L}$ C-reactive protein, CRP, normal value $<5 \mathrm{mg} / \mathrm{l}$ ), and a severe hyponatremia of $107 \mathrm{mmol} / \mathrm{l}$ (normal value between 135-145 mmol/l). No pathological findings were seen on the computed tomography scan of the abdomen, especially no abscess or perforation. The results from a lumbar puncture were inconspicuous and ruled out an infection or inflammation.

The severity of the diarrhea as well as inflammatory markers (CRP max $543 \mathrm{mg} / \mathrm{L}$, procalcitonin, $84.88 \mu \mathrm{g} / \mathrm{L}$, normal value $<0.1 \mu \mathrm{g} / \mathrm{L}$ ) increased quickly despite management in the intermediate care unit. The patient now lost up to $12 \mathrm{~L}$ stool per day and was admitted to the intensive care unit for further treatment. On gross examination, her stool was brown and watery. Cultures of the blood, urine and stool were collected and an empiric antibiotic treatment was initiated. With a suspected gastrointestinal focus antibiotic treatment consisted of piperacillin-tazobactam i.v. (4.5 g every $8 \mathrm{~h}$ ) and vancomycin p.o. (250 mg every $6 \mathrm{~h}$ ). The blood and urine cultures did not detect any bacterial growth. A molecular multiplex real-time RT-PCR test for detection of numerous gastrointestinal pathogens (QIAstat-Dx Gastrointestinal (GI) panel, QIAGEN, Hilden, Germany) performed directly from the stool was negative (Table 1). The Clostridioides difficile glutamate dehydrogenase (GDH) antigen in the stool was positive, as tested by VIDAS $C$. difficile GDH assay (bioMerieux, France), but the toxin genes remained negative as tested by real-time PCR (GeneXpert, Xpert C. difficile BT assay, Cepheid, USA).

After 3 days of treatment without any improvement in the patient's condition, the piperacillin-tazobactam and vancomycin was stopped and meropenem i.v ( $1 \mathrm{~g}$ every $8 \mathrm{~h}$ ) and metronidazol i.v. (500 mg every 8 h) was started instead.

For bacterial culture, the stool was incubated on MacConkey's agar (Oxoid, UK), Columbia 5\% sheep blood agar (bioMérieux, Marcy l'Etoile, France) and deoxycholate citrate agar (DCA, Oxoid, UK) at $37{ }^{\circ} \mathrm{C}$. After $24 \mathrm{~h}$, bacterial growth appeared on the plates showing yellow sucrose fermenting colonies on the DCA plate. The colonies were non-lactose fermenting on DCA and MacConkey's agar. On the sheep blood agar, bacterial colonies showed a remarkable $\beta$-hemolysis. The catalase and oxidase tests both were positive.

The bacteria were identified as $A$. hydrophila by the matrix-assisted laser desorption ionization-time of flight mass spectrometry (MALDI-TOF MS, Bruker Daltonik, Bremen, Germany; using the MALDI Biotyper version 7.0). The antimicrobial susceptibility testing was performed by disk diffusion test on Mueller-Hinton agar plates $(\mathrm{MH}$, Becton Dickinson, Franklin Lakes, NJ) and revealed susceptibility to meropenem, cefepime, piperacillin-tazobactam, ceftriaxone, 
Table 1 Pathogen targets of the QIAstat-Dx Gastrointestinal panel (QIAGEN)

\begin{tabular}{lll}
\hline Bacterial targets & Viruses & Parasites \\
\hline Clostridioides difficile toxin A/B & Adenovirus F40/41 & Cryptosporidium spp. \\
Enteroaggregative E. coli (EAEC) & Astrovirus & Cyclospora cayetanensis \\
Enteropathogenic E. coli (EPEC) & Norovirus GI & Entamoeba histolytica \\
Enterotoxigenic E. coli (ETEC) & Norovirus GII & Giardia lamblia \\
Enteroinvasive E. coli (ElEC)/Shigella & Sapovirus A $(G l$, GII, GIV, GV) & \\
Shiga-like toxin-producing E. coli (STEC) & & \\
Shiga toxin-producing E. coli (STEC) O157:H7 & & \\
Campylobacter spp. & & \\
Plesiomonas shigelloides & & \\
Salmonella spp. & & \\
Vibrio cholera & & \\
Vibrio parahaemolyticus & & \\
Vibrio vulnificus & & \\
Yersinia enterocolitica & & \\
\hline
\end{tabular}

Table 2 Antimicrobial susceptibility testing of the A. hydrophila strain

\begin{tabular}{|c|c|c|c|c|c|c|}
\hline \multirow[t]{2}{*}{ Antimicrobial agent } & \multirow[t]{2}{*}{ Disk content $(\mu \mathrm{g})$} & \multirow[t]{2}{*}{$\begin{array}{l}\text { Zone diameter } \\
(\mathrm{mm})\end{array}$} & \multicolumn{3}{|c|}{$\begin{array}{l}\text { Interpretive categories and zone } \\
\text { diameter breakpoints }{ }^{\mathrm{a}}(\mathrm{mm})\end{array}$} & \multirow[t]{2}{*}{ Interpretation } \\
\hline & & & $\mathrm{S}$ & I & $\mathbf{R}$ & \\
\hline Ampicillin & 10 & 6 & n.a & n.a & n.a & Resistant \\
\hline Amoxicillin-clavulanate & $20 / 10$ & 12 & n.a & n.a & n.a & Resistant \\
\hline Piperacillin-tazobactam & $100 / 10$ & 23 & $\geq 21$ & $18-20$ & $\leq 17$ & Susceptible \\
\hline Ceftriaxone & 30 & 37 & $\geq 23$ & $20-22$ & $\leq 19$ & Susceptible \\
\hline Cefepime & 30 & 33 & $\geq 25$ & $19-24$ & $\leq 18$ & Susceptible \\
\hline Meropenem & 10 & 27 & $\geq 23$ & $20-22$ & $\leq 19$ & Susceptible \\
\hline Nalidixic acid & 30 & 31 & n.a & n.a & n.a & Susceptible \\
\hline Trimethoprim-sulfamethoxazole & $1.25 / 23.75$ & 22 & $\geq 16$ & $11-15$ & $\leq 10$ & Susceptible \\
\hline Amikacin & 30 & 21 & $\geq 17$ & $15-16$ & $\leq 14$ & Susceptible \\
\hline
\end{tabular}

$S$ susceptible, I intermediate, $R$ resistant, n.a. not available

${ }^{\text {a }}$ According to Clinical and Laboratory Standards Institute guidelines, M45 3rd edition
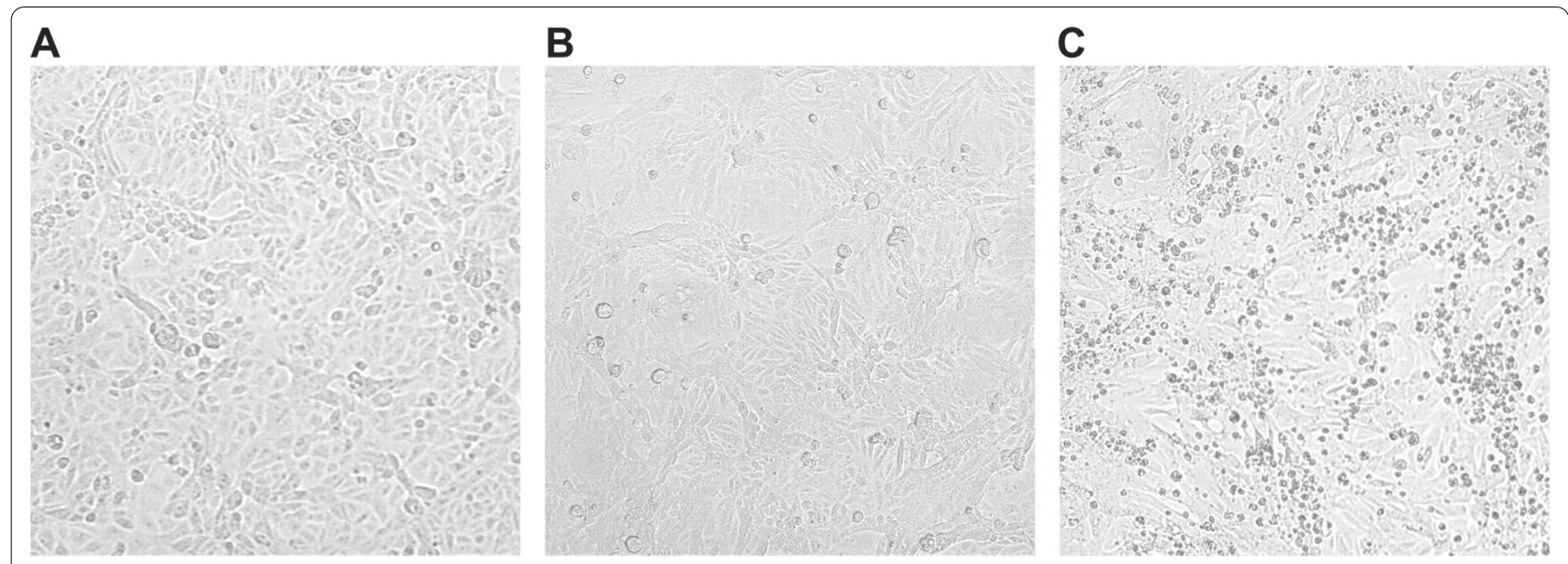

Fig. 1 Cytotoxic effects of $A$. hydrophila cultured supernatant in Vero cells $\mathbf{A}$ negative control with culture media, $\mathbf{B}$ cytotoxic damage of $A$. hydrophila after 1 day incubation exhibiting cell rounding $\mathbf{C}$ cytotoxic damage of $\mathbf{A}$. hydrophila after $\mathbf{4}$ days incubation, indicating cellular vacuolation and monolayer destruction 
nalidixic acid, trimethoprim-sulfamethoxazole and amikacin (Table 2). The strain was resistant to amoxicillin and amoxicillin-clavulanate (Table 2) Interpretative criteria according to the Clinical and Laboratory Standards Institute guidelines, M45 3rd edition, were applied.

After the identification of A. hydrophila, metronidazol was stopped and following a 10-day treatment with meropenem and intravenous fluid substitution, the patient recovered slowly and hospital discharge was possible. During the next visit, four weeks after discharge, the patient presented without fever or abdominal pain and the frequency of stool was again 3 to 4 times per day.

To demonstrate whether the clinical A. hydrophila strain produced an enterotoxin, the A. hydrophila culture supernatant was analyzed for enterotoxic activity in cell cultures [22, 23]. The bacterial strain was cultivated in trypticase soy broth media (Becton Dickinson, USA). The culture supernatant was sterile filtered and inoculated in a confluent monolayer of Vero (African green monkey kidney) cell lines cultivated in tissue culture tubes with Eagle's minimum essential medium (MEM, Dulbecco's, bioswisstec AG, Schaffhausen, Switzerland). Then the tube was incubated at $37^{\circ} \mathrm{C}$ in a $5 \% \mathrm{CO}_{2}$ incubator. Trypticase soy broth and MEM medium were used as negative controls. Cell monolayer morphology was observed using an inverted microscope. After 1 day, morphological alterations in Vero cells were observed inducing rounding, detachment, cellular vacuolation and monolayer destruction (Fig. 1). These observations were consistent with the alterations found in previous reports [22-24]. The negative culture controls did not show these modifications and displayed a confluent monolayer (Fig. 1). The presence of an enterotoxin with cytotoxic activity was suggested.

\section{Discussion and conclusion}

We reported a case of severe gastroenteritis due to $A$. hydrophila in a patient with an anti-Hu syndrome. The patient had chronic diarrhea after colectomy, which was exacerbated requiring intensive care. In the literature, severe gastroenteritis caused by Aeromonas spp. are described in immunocompromised individuals [25] and in patients with chronic inflammatory bowel disease [26]. In our case, the patient neither received immunosuppressive therapy nor other medications, which would increase the patient's vulnerability to severe infection. The common differential diagnoses and other pathogens were excluded. To our knowledge, this is the first case of severe gastroenteritis due to $A$. hydrophila in a patient with anti-Hu syndrome.
Despite the association of anti-Hu antibodies with paraneoplastic syndromes, we did not find any underlying tumour in our patient. In a study by Graus et al., a number of patients with neurological paraneoplastic syndromes and anti-Hu antibody positivity in the absence of any tumour were described [27]. In patients such as our patient, with chronic pseudo-obstructions and after ileocoecal surgery, there is a change in the gastrointestinal microbiome $[28,29]$ and intestinal bacterial overgrowth is more prevalent [30,31]. Additionally, there is some evidence concerning an increased susceptibility to gastrointestinal infections in patients with a history of gut surgery [32]. Thus, chronic intestinal pseudo-obstructions might be a possible explanation for our patient's predisposition to severe gastroenteritis with $A$. hydrophila.

Although Aeromonas spp. most commonly are isolated in the gastrointestinal tract, their role as an enteropathogen is still controversial [19]. The asymptomatic colonization in developed countries range from 0 to $4 \%$ while the isolation rate from stool in persons with diarrheal illness ranges from 0.8 to $7.4 \%$ [16, 33]. Nevertheless, there are many case reports that describe Aeromonas spp. as a causative enteropathogen [2]. In these reports, either an isolation of the microorganism in the feces or tissue samples was achieved or a positive serological response was present [2].

Beyond any doubt, evidence for local outbreaks is not sufficient, the literature is controversial $[34,35]$. In our patient, the source of the infection was not found. Aeromonas spp. are environmental opportunistic pathogens that are inhabitants of aquatic ecosystems such as groundwater but might be present also in drinking water or dairy products [36].

In our case, bacterial stool cultures showed growth of $A$. hydrophila. In contrast, a fast multiplex RT-PCR covering a large panel of common gastrointestinal pathogens performed directly from the stool specimen remained negative. In the last years, numerous multiplex systems covering a broad range of gastrointestinal pathogens including bacteria, viruses and parasites appeared on the market. One such system is the QIAstat-Dx GI panel (QIAGEN), which is highlighted by a short turnaround time and was demonstrated to be a valuable tool for diagnosis of gastrointestinal pathogens [37]. Despite the advantages of molecular-based syndromic stool pathogen panels, rare pathogens such as Aeromonas spp. are not covered in most syndromic assays including the QIAstat-Dx GI panel [7] (Table 1). Because many laboratories are likely adopting these multiplex syndromic panels and no longer performing stool cultures, gastroenteritis caused by Aeromonas sp. might be underestimated. 
In our patient, the treatment with piperacillin-tazobactam was not successful in alleviating the symptoms despite proven susceptibility in vitro. A possible explanation might be the presence of chromosomally mediated $\beta$-lactamases such as the AmpC $\beta$-lactamase, missed by conventional phenotypic tests [38]. In a Korean study analyzing bacteremia caused by Aeromonas spp., cases were observed with a piperacillin-tazobactam resistance [39]. When meropenem was installed, clinical improvement and laboratory response were observed. Nevertheless, the use of meropenem is controversial in the treatment of Aeromonas spp. due to the possibility of existing chromosomally mediated CphA carbapenemases [38]. Recent reports have identified carbapenemase-producing Aeromonas spp. strains in clinical specimens [40].

The production of a cytotoxic enterotoxin is an important virulence factor of $A .{ }^{\circ}$ hydrophila [10, 22, 41]. The presence of the cytotoxic enterotoxin (Act protein) in clinical $A$. ${ }^{\circ}$ ydrophila strains was previously shown to be associated with cytotoxicity in Vero cells thus indicating the potential of causing severe infections [42]. We have demonstrated the cytotoxic effect of the supernatant of the clinical A. hydrophila strain in Vero cells (Fig. 1). Therefore, we speculate that the clinical $A$. hydrophila strain was a cytotoxic enterotoxin producing strain, which was the cause for the severe clinical presentation. A limitation of our case report is, that we have not purified the toxin from the clinical $A$. hydrophila isolate and we have not proved the presence of the act gene, which encodes the cytotoxic enterotoxin, by molecular methods.

Although in some gastroenteritis cases the role of the isolation of Aeromonas spp. in stool specimens is discussed controversially, in our case, we have proof of the cytotoxic effect of the supernatant of the isolated $A$. hydrophila strain indicative of the presence of an enterotoxin and a remarkable clinical improvement in the patient's condition after instalment of meropenem. The patient had a decrease in stool frequency, resolution of fever and the inflammatory parameter decreased significantly.

Aeromonas spp. should be considered in the differential diagnosis of acute gastroenteritis, which revealed broad spectrum multiplex-PCR negative results. Early diagnosis and initiation of appropriate therapy is crucial for the clinical management. This case might serve as an argument that clinicians should consider also rare causative agents of gastroenteritis and highlights the need to perform culture methods in PCR-negative tested stool specimens where a clinical suspicion of gastrointestinal infection exists.
Abbreviations

CRP: C-reactive protein; MEM: Minimum essential medium; QIAstat-Dx Gl panel: QIAstat-Dx Gastrointestinal panel.

\section{Acknowledgements}

We thank the technicians of the Institute of Medical Microbiology for their dedicated help and the Institute of Medical Microbiology, University of Zurich, for continuous support.

\section{Authors' contributions}

MG and RZ designed the plan of the work. MG, DP and AA acquired the clinical data and analyzed the clinical features. MG wrote the first draft, compiled the patient's medical history and laboratory data. MG, AZ and RZ acquired and interpreted the laboratory findings. AZ performed the toxin experimental part. AZ critically reviewed the manuscript and substantively revised the work. All authors participated in writing the manuscript. All authors read and approved the manuscript.

\section{Funding}

The authors received no financial support for the research, authorship and/or publication of this article.

\section{Availability of data and materials}

All data generated or analysed during this study are included in this published article.

\section{Declarations}

Ethics approval and consent to participate

Not applicable.

\section{Consent for publication}

Written informed consent was obtained from the patient for publication of this case report.

\section{Competing interests}

There are no conflicts of interest to declare.

\section{Author details}

${ }^{1}$ Institute of Medical Microbiology, University of Zurich, Gloriastrasse 28/30, 8006 Zurich, Switzerland. ' Division of Infectious Diseases and Hospital Epidemiology, University of Zurich, University Hospital of Zurich, Zurich, Switzerland. ${ }^{3}$ Division of Gastroenterology, University of Zurich, University Hospital of Zurich, Zurich, Switzerland.

Received: 19 May 2021 Accepted: 12 October 2021

Published online: 24 October 2021

\section{References}

1. Abbott SL, Cheung WK, Janda JM. The genus Aeromonas: biochemical characteristics, atypical reactions, and phenotypic identification schemes. J Clin Microbiol. 2003;41(6):2348-57.

2. Janda JM, Abbott SL. Evolving concepts regarding the genus Aeromonas: an expanding Panorama of species, disease presentations, and unanswered questions. Clin Infect Dis. 1998;27(2):332-44.

3. Vila J, et al. Aeromonas spp. and traveler's diarrhea: clinical features and antimicrobial resistance. Emerg Infect Dis. 2003;9(5):552-5.

4. Janda JM, Abbott SL. The genus Aeromonas: taxonomy, pathogenicity, and infection. Clin Microbiol Rev. 2010;23(1):35-73.

5. Hanninen ML, et al. Association of Aeromonas spp. with travellers' diarrhoea in Finland. J Med Microbiol. 1995;42(1):26-31.

6. Yamada S, et al. Incidence and clinical symptoms of Aeromonas-associated travellers' diarrhoea in Tokyo. Epidemiol Infect. 1997;119(2):121-6.

7. Schuetz AN. Emerging agents of gastroenteritis: Aeromonas, Plesiomonas, and the diarrheagenic pathotypes of Escherichia coli. Semin Diagn Pathol. 2019:36(3):187-92. 
8. van Zwetselaar M, et al. Aeromonas caviae mimicking Vibrio cholerae infectious enteropathy in a cholera-endemic region with possible public health consequences: two case reports. J Med Case Rep. 2018;12(1):71.

9. Clinical relevance of Aeromonas sM503. 2006. 17(3): 91.

10. Chopra AK, Houston CW. Enterotoxins in Aeromonas-associated gastroenteritis. Microbes Infect. 1999;1(13):1129-37.

11. Thornley JP, et al. Virulence properties of clinically significant Aeromonas species: evidence for pathogenicity. Rev Med Microbiol. 1997;8(2):61-72.

12. Barnett TC, et al. Aeromonas spp. possess at least two distinct type IV pilus families. Microb Pathog. 1997;23(4):241-7.

13. Grim, CJ, et al. Characterization of Aeromonas hydrophila wound pathotypes by comparative genomic and functional analyses of virulence genes. MBio, 2013; 4(2): e00064.

14. Sha J, Kozlova EV, Chopra AK. Role of various enterotoxins in Aeromonas hydrophila-induced gastroenteritis: generation of enterotoxin genedeficient mutants and evaluation of their enterotoxic activity. Infect Immun. 2002;70(4):1924-35.

15. Ferguson MR, et al. Hyperproduction, purification, and mechanism of action of the cytotoxic enterotoxin produced by Aeromonas hydrophila. Infect Immun. 1997:65(10):4299-308.

16. Albert MJ, et al. Prevalence of enterotoxin genes in Aeromonas spp. isolated from children with diarrhea, healthy controls, and the environment. J Clin Microbiol, 2000; 38(10): 3785-90.

17. Chopra AK, et al. Molecular and biochemical characterization of a heatlabile cytotonic enterotoxin from Aeromonas hydrophila. Microb Pathog. 1996;21(5):357-77.

18. Brown RL, Sanderson K, Kirov SM. Plasmids and Aeromonas virulence. FEMS Immunol Med Microbiol. 1997;17(4):217-23.

19. von Graevenitz A. The role of Aeromonas in diarrhea: a review. Infection. 2007;35(2):59-64.

20. Senties-Madrid H, Vega-Boada F. Paraneoplastic syndromes associated with anti-Hu antibodies. Isr Med Assoc J. 2001;3(2):94-103.

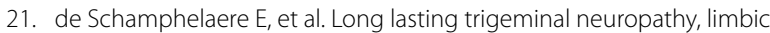
encephalitis and abdominal ganglionitis without primary cancer: an atypical case of Hu-antibody syndrome. Clin Neurol Neurosurg, 2020; 194 : 105849.

22. Lopes AC, et al. Diarrhea outbreak in pernambuco, brazil, associated with a heat-stable cytotoxic enterotoxin produced by Aeromonas caviae. Rev Inst Med Trop Sao Paulo. 2015;57(4):349-51.

23. Konowalchuk J, Speirs JI, Stavric S. Vero response to a cytotoxin of Escherichia coli. Infect Immun. 1977;18(3):775-9.

24. Palma-Martinez I, et al. Active Shiga-Like Toxin Produced by Some Aeromonas spp., Isolated in Mexico City. Front Microbiol. 2016; 7: 1522.

25. Liao WC, Cappell MS. Treatment with ciprofloxacin of Aeromonas hydrophila associated colitis in a male with antibodies to the human immunodeficiency virus. J Clin Gastroenterol. 1989;11(5):552-4.

26. Lobatón T, et al. Aeromonas species: an opportunistic enteropathogen in patients with inflammatory bowel diseases? A single center cohort study. Inflamm Bowel Dis. 2014;21(1):71-8.
27. Graus F, et al. Anti-Hu-associated paraneoplastic encephalomyelitis: analysis of 200 patients. Brain. 2001;124(Pt 6):1138-48.

28. Devine AA, et al. Impact of ileocecal resection and concomitant antibiotics on the microbiome of the murine jejunum and colon. PLOS ONE, 2013; 8(8): e73140.

29. Hegde $\mathrm{S}$, et al. Microbiota dysbiosis and its pathophysiological significance in bowel obstruction. Sci Rep. 2018:8(1):13044.

30. Sachdev AH, Pimentel M. Gastrointestinal bacterial overgrowth: pathogenesis and clinical significance. Ther Adv Chronic Dis. 2013;4(5):223-31.

31. Dukowicz AC, Lacy BE, Levine GM. Small intestinal bacterial overgrowth: a comprehensive review. Gastroenterol Hepatol (N Y). 2007;3(2):112-22.

32. Napolitano LM, Edmiston CE Jr. Clostridium difficile disease: Diagnosis, pathogenesis, and treatment update. Surgery. 2017;162(2):325-48.

33. Svenungsson $B$, et al. Enteropathogens in adult patients with diarrhea and healthy control subjects: a 1-year prospective study in a Swedish clinic for infectious diseases. Clin Infect Dis. 2000;30(5):770-8.

34. Edberg SC, Browne FA, Allen MJ. Issues for microbial regulation: Aeromonas as a model. Crit Rev Microbiol. 2007;33(1):89-100.

35. Krovacek K, et al. Isolation, and virulence profiles, of Aeromonas hydrophila implicated in an outbreak of food poisoning in Sweden. Microbiol Immunol. 1995;39(9):655-61.

36. Khajanchi BK, et al. Distribution of virulence factors and molecular fingerprinting of Aeromonas species isolates from water and clinical samples: suggestive evidence of water-to-human transmission. Appl Environ Microbiol. 2010;76(7):2313-25.

37. Boers SA, et al. Multicenter Evaluation of QIAstat-Dx Respiratory Panel V2 for Detection of Viral and Bacterial Respiratory Pathogens. J Clin Microbiol, 2020; 58: 6.

38. Chen PL, Ko WC, Wu CJ. Complexity of beta-lactamases among clinical Aeromonas isolates and its clinical implications. J Microbiol Immunol Infect. 2012;45(6):398-403.

39. Rhee JY, Jung DS, Peck KR. Clinical and Therapeutic Implications of Aeromonas Bacteremia: 14 Years Nation-Wide Experiences in Korea. Infect Chemother. 2016;48(4):274-84.

40. Sinclair HA, et al. Genotypic and phenotypic identification of Aeromonas species and CphA-mediated carbapenem resistance in Queensland Australia. Diagn Microbiol Infect Dis. 2016;85(1):98-101.

41. Cumberbatch $\mathrm{N}$, et al. Cytotoxic enterotoxin produced by Aeromonas hydrophila: relationship of toxigenic isolates to diarrheal disease. Infect Immun. 1979;23(3):829-37.

42. Ottaviani $D$, et al. Putative virulence properties of Aeromonas strains isolated from food, environmental and clinical sources in Italy: a comparative study. Int J Food Microbiol. 2011;144(3):538-45.

\section{Publisher's Note}

Springer Nature remains neutral with regard to jurisdictional claims in published maps and institutional affiliations.

\footnotetext{
Ready to submit your research? Choose BMC and benefit from:

- fast, convenient online submission

- thorough peer review by experienced researchers in your field

- rapid publication on acceptance

- support for research data, including large and complex data types

- gold Open Access which fosters wider collaboration and increased citations

- maximum visibility for your research: over $100 \mathrm{M}$ website views per year
}

At BMC, research is always in progress.

Learn more biomedcentral.com/submissions 